\title{
Percolation for Low Energy Clusters and Discrete Symmetry Breaking in Classical Spin Systems
}

\author{
Hans-Otto Georgii ${ }^{1}$
}

Fakultät für Mathematik, Abt. 1, Universität Bielefeld, D-4800 Bielefeld, Federal Republic of Germany

\begin{abstract}
We consider classical lattice systems in two or more dimensions with general state space and with short-range interactions. It is shown that percolation is a general feature of these systems: If the temperature is sufficiently low, then almost surely with respect to some equilibrium state there is an infinite cluster of spins trying to form a ground state. For systems having several stable sets of symmetry-related ground states we show that at low temperatures spontaneous symmetry breaking occurs because in a twodimensional subsystem there is a unique infinite cluster of this type.
\end{abstract}

\section{Introduction}

Percolation theory has recently been successfully used in analyzing the properties of the two-dimensional Ising model $[1,12]$. Percolation is also implicitly involved in the well-known Peierls argument for the existence of phase transitions. Here we introduce the notion of "low energy clusters". This concept enables us to show that percolation at low temperatures is a general phenomenon for spin systems in two or more dimensions. Under certain circumstances this kind of percolation automatically implies the existence of several distinct equilibrium states; this gives an intuitively appealing picture of the mechanism which leads to a spontaneous breaking of discrete symmetries. (This picture fails to explain certain phenomena in three dimensions such as the breaking of continuous symmetries and the existence of nontranslation invariant Gibbs states in the Ising model.)

Passing on to precise definitions, let $d \geqq 2, L=\mathbb{Z}^{d}$ (the integer lattice), and consider a system of spins on $L$ taking values in a probability space $(E, \mathscr{E}, \lambda)$. The spins interact via a nearest-neighbour potential which is a measurable symmetric function

$$
\varphi: E \times E \rightarrow \mathbb{R},
$$

satisfying

$$
m \equiv \inf \varphi(\cdot, \cdot)>-\infty .
$$

1 Present address: Mathematisches Institut der Universität, Theresienstraße 39, D-8000 München 2, Federal Republic of Germany 
So the formal Hamiltonian reads

$$
H(\sigma)=\sum_{\{i, j\}} \varphi\left(\sigma_{i}, \sigma_{j}\right)
$$

here $\sigma=\left(\sigma_{i}\right)_{i \in L} \in E^{L}$, and the sum extends over all pairs of adjacent sites in $L$. For each $\beta \geqq 0$ we let $(5(\beta)$ denote the set of all Gibbs measures (equilibrium states) on $(E, \mathscr{E})^{L}$ with respect to $\beta \varphi$ and the reference measure $\lambda$ (the precise definition is given below).

Fix $\varepsilon>0$. For each $\sigma \in E^{L}$ we construct a graph $g_{\varepsilon}(\sigma)$ with vertex set $L$ by drawing an edge between any two adjacent sites for which $\sigma_{i}$ and $\sigma_{j}$ have a low interaction energy in the sense that

$$
\varphi\left(\sigma_{i}, \sigma_{j}\right) \leqq m+\varepsilon
$$

We are interested in the event

$$
\left\{\exists c_{\infty}^{\varepsilon}\right\}
$$

consisting of all $\sigma \in E^{L}$ for which $g_{\varepsilon}(\sigma)$ contains an infinite cluster (i.e., an infinite connected component). Our question is: For which $\beta$ can one find a Gibbs measure $\mu \in \mathfrak{5}(\beta)$ such that

$$
\mu\left(\exists c_{\infty}^{\varepsilon}\right)>0 ?
$$

As an example let us consider the plane rotator model in dimension $d=2$. Here $E=S^{1}$ (the unit circle in the plane), $\lambda$ is the normalized Haar measure, and

$$
\varphi(x, y)=-x \cdot y .
$$

Thus $m=-1$, and (1.3) holds iff $\sigma_{i}$ and $\sigma_{j}$ have nearly the same orientation. So in this case our question reduces to the study of what could be called "small deviation clusters". It is known $[3,15]$ in this model that for each $\beta \geqq 0$ there is only one measure in $\mathfrak{5}(\beta)$ which is invariant under translations, and this measure is also invariant under simultaneous rotation of all spins. In other words: in contrast to the case $d=3$ [9], no symmetry breaking occurs. Therefore one is interested in the question of whether this system exhibits some weaker form of ordering. S. Miracle-Sole (private communication) conjectured that infinite "small deviation clusters" occur. A positive answer to this is implied by the following result, which will be proved below under mild topological assumptions: If $\beta$ is sufficiently large then there is some translation invariant Gibbs measure $\mu \in \mathfrak{G}(\beta)$ such that

$$
\mu\left(\exists c_{\infty}^{\varepsilon}\right)=1 .
$$

The proof of this result employs ideas similar to those of Shlosman [19] and makes use of reflection positivity and chessboard estimates $[7,8,9]$. We will actually prove the stronger assertion that any given two-dimensional sublattice of $L$ contains a unique infinite low energy cluster. Moreover, we will consider a slightly more general setup which includes systems with next-nearest neighbour interactions. Section 2 contains the precise formulation of our results on percolation; these will be proved in Sect. 4. In Sect. 3 we show that the existence of a unique infinite low energy cluster provides a simple and appealing approach to the study of 
symmetry breakdown. This approach consists in a distillation of the three ingredients which make up the well-known Peierls argument: percolation, symmetry, and stability of the ground states, and will be illustrated by a series of examples already known to exhibit a phase transition.

We conclude this introduction by giving examples which show that infinite low energy clusters need not occur when both $\varepsilon$ and $\beta$ are small.

(1.7) Example. The two-dimensional Ising model.

Here $d=2, E=\{-1,1\}, \lambda$ is normalized counting measure, and $\varphi$ is given by

$$
\varphi(x, y)=-x y .
$$

Thus $m=-1$, and for $\varepsilon<2$ we have

$$
\varphi(x, y) \leqq m+\varepsilon \text { iff } x=y .
$$

Therefore each infinite cluster of $g_{\varepsilon}(\cdot)$ is either a $(+)$ cluster or a $(-)$ cluster (in the obvious sense). Thus the results of $[4,1,12]$ show that when $\beta \leqq \beta_{c} \equiv \frac{1}{2} \sinh ^{-1} 1$ then

$$
\mu\left(\exists c_{\infty}^{\varepsilon}\right)=0
$$

for the (unique) measure $\mu \in \mathfrak{G}(\beta)$, while on the other hand

$$
\mu\left(\exists c_{\infty}^{\varepsilon}\right)=1 \text { for all } \mu \in \mathfrak{b}(\beta)
$$

when $\beta>\beta_{c}$.

Further examples are provided by the following proposition which applies to continuous models such as, for instance, the plane rotator model.

(1.8) Proposition. Suppose $\varphi$ is bounded and satisfies

$$
\lambda^{2}(\varphi(\cdot, \cdot)=m)=0 .
$$

Then there is a function $\beta(\cdot) \geqq 0$ such that

$$
\beta(\varepsilon) \uparrow \infty \text { as } \varepsilon \downarrow 0
$$

and

$$
\sup _{\mu \in(\mathfrak{G}(\beta)} \mu\left(\exists c_{\infty}^{\varepsilon}\right)=0 \text { when } 0 \leqq \beta<\beta(\varepsilon) .
$$

This will be proved at the end of Sect. 4 .

\section{Percolation for Low Energy Clusters}

The study of low energy clusters as described above is a bond percolation problem. However, it will be convenient to consider a more general site percolation problem on the dual lattice which allows us to also study systems with next-nearest neighbour interactions. The dual of $L$ is

$$
L^{*}=L+\left(\frac{1}{2}, \ldots, \frac{1}{2}\right)
$$

Each $a \in L^{*}$ is identified with the elementary cube consisting of the $2^{d}$ sites of $L$ 
with distance $\sqrt{d / 2}$ from $a$, i.e.

$$
a \equiv\left\{i \in L: i=a+\left( \pm \frac{1}{2}, \ldots, \pm \frac{1}{2}\right)\right\} .
$$

We let $v \equiv\left(\frac{1}{2}, \ldots, \frac{1}{2}\right)$ denote the elementary cube at the origin of $L^{*}$.

Next let $(E, \mathscr{E}, \lambda)$ be a probability space. We will assume that $E$ is a Polish space and $\mathscr{E}$ its Borel $\sigma$-algebra. Without loss of generality we will further assume that $\lambda$ charges every nonempty open subset of $E$. The configuration space is

$$
(\Omega, \mathscr{F})=(E, \mathscr{E})^{L} .
$$

For each $\Lambda \subset L$ we consider the sub- $\sigma$-algebra

$$
\mathscr{F}_{\Lambda}=\mathscr{E}^{\Lambda} \otimes\{E, \varnothing\}^{L \backslash \Lambda}
$$

of $\mathscr{F}$. If $\sigma=\left(\sigma_{i}\right)_{i \in L} \in \Omega$ then $\sigma_{\Lambda}=\left(\sigma_{i}\right)_{i \in \Lambda}$ is the restriction of $\sigma$ to $\Lambda$; if $\sigma, \tau \in \Omega$ then $\tau_{\Lambda} \sigma_{L \backslash \Lambda}$ is the configuration on $L$ which coincides with $\tau$ on $\Lambda$ and with $\sigma$ on $L \backslash \Lambda$. The translation group $\left(\theta_{i}\right)_{i \in L}$ acting on $\Omega$ is defined by

$$
\left(\theta_{i} \sigma\right)_{j}=\sigma_{j-i} \quad(i, j \in L, \sigma \in \Omega) .
$$

We consider formal Hamiltonians of the form

$$
H(\sigma)=\sum_{a \in L^{*}} \Phi(a, \sigma) ;
$$

here $\Phi: L^{*} \times \Omega \rightarrow \mathbb{R}$ is assumed to have the following properties:

(2.2) There is a function $\Phi_{v}: E^{v} \rightarrow \mathbb{R}$ with

$$
\Phi(v, \sigma)=\Phi_{v}\left(\sigma_{v}\right) \text { for all } \sigma \in \Omega .
$$

(2.3) Translation invariance. For all $a \in L^{*}, i \in L, \sigma \in \Omega$

$$
\Phi(a, \sigma)=\Phi(a+i, \theta, \sigma) \text {. }
$$

(2.4) Reflection invariance. For all $s \in E^{v}$ and $1 \leqq k \leqq d$

$$
\Phi_{v}\left(r_{k} s\right)=\Phi_{v}(s)
$$

here $r_{k}$ is the reflection of $v$ with respect to the hyperplane

(2.5) $\Phi_{v}$ is continuous.

$$
\left\{x=\left(x_{1}, \ldots, x_{d}\right) \in \mathbb{R}^{d}: x_{k}=\frac{1}{2}\right\} .
$$

$$
m \equiv \inf \Phi_{v}>-\infty .
$$

Clearly the Hamiltonian (1.2) can be written in the form (2.1): it is sufficient to put

$$
\Phi_{v}(s)=2^{1-d} \sum_{\{i, j\} \subset v} \varphi\left(s_{i}, s_{j}\right) .
$$

Therefore the present framework includes that of the introduction.

For $\beta \geqq 0$ we let $(\mathfrak{S}(\beta)$ denote the set of all Gibbs measures (Dobrushin, Lanford and Ruelle or DLR states) for $\beta \Phi$ and $\lambda$; these are all probability measures $\mu$ on $(\Omega, \mathscr{F})$ such that for all finite nonempty subsets $\Lambda$ of $L$ and all $A \in \mathscr{F}_{\Lambda}$

$$
\mu\left(A \mid \mathscr{F}_{L \backslash \Lambda}\right)(\sigma)=\gamma^{\beta}(A \mid \sigma) \equiv \int_{A} \lambda^{L}(d \tau) \exp \left[-\beta H_{\Lambda}(\tau \mid \sigma)\right] / Z_{\Lambda}^{\beta}(\sigma)
$$


here

$$
H_{\Lambda}(\tau \mid \sigma)=\sum_{a \in L^{*}: a \cap \Lambda \neq \varnothing} \Phi\left(a, \tau_{\Lambda} \sigma_{L \backslash \Lambda}\right)
$$

and

$$
0<Z_{\Lambda}^{\beta}(\sigma) \equiv \int \lambda^{L}(d \tau) \exp \left[-\beta H_{\Lambda}(\tau \mid \sigma)\right]<\infty
$$

due to (2.6). We are particularly interested in Gibbs measures which are limits of finite volume Gibbs distributions with periodic boundary conditions: Let $n \geqq 1$ and

$$
\Lambda=\Lambda_{n}=\left\{i=\left(i_{1}, \ldots, i_{d}\right) \in L:-n<i_{k} \leqq n \text { for } 1 \leqq k \leqq d\right\} .
$$

We view $\Lambda$ as a torus by considering $i, j \in \Lambda$ as nearest neighbours iff $i_{k} \equiv j_{k} \pm 1$ $\bmod 2 n$ and $i_{k^{\prime}}=j_{k^{\prime}}$ for $k^{\prime} \neq k$ for some $1 \leqq k \leqq d$. Accordingly we identify each $a \in \Lambda^{*}=\Lambda+\left(\frac{1}{2}, \ldots, \frac{1}{2}\right)$ with the elementary cube

$$
\left\{i \in \Lambda: i=a+\left( \pm \frac{1}{2}, \ldots, \pm \frac{1}{2}\right) \bmod 2 n\right\} \text {. }
$$

If $\sigma \varepsilon E^{\Lambda}$ and $a \in \Lambda^{*}$ then $\Phi(a, \sigma)$ is defined in the natural way. The Gibbs distribution in $\Lambda$ for $\beta \Phi$ with periodic boundary condition is given by

$$
\gamma_{\Lambda}^{\beta}(d \sigma)=\exp \left[-\beta H_{\Lambda}(\sigma)\right] \lambda^{\Lambda}(d \sigma) / Z_{\Lambda}^{\beta} ;
$$

here

$$
H_{\Lambda}(\sigma)=\sum_{a \in \Lambda^{*}} \Phi(a, \sigma)
$$

and the normalizing constant $Z_{\Lambda}^{\beta}$ is finite due to (2.6). We let $\mathfrak{G}_{0}(\beta)$ denote the set of all probability measures on $(\Omega, \mathscr{F})$ which are weak limit points of the sequence $\gamma_{\Lambda_{n}}^{\beta}, n \rightarrow \infty$. It follows easily from $(2.5)$ that $\mathfrak{G}_{0}(\beta) \subset \mathfrak{G}(\beta)$. We assume $\mathfrak{G}_{0}(\beta) \neq \varnothing$ for all $\beta \geqq 0$; of course this will always be true when $E$ is compact.

Now we introduce low energy site percolation on $L^{*}$. Fix an $\varepsilon>0$. For each $\sigma \in \Omega$ we form a subgraph $G_{\varepsilon}(\sigma)$ of $L^{*}$ consisting of the vertex set

$$
V_{\varepsilon}(\sigma)=\left\{a \in L^{*}: \Phi(a, \sigma) \leqq m+\varepsilon\right\}
$$

and with edges connecting any pair $\{a, b\}$ of adjacent sites in $V_{\varepsilon}(\sigma)$; here $a$ and $b$ are said to be adjacent if Euclidean distance between them is 1 or, equivalently, if the corresponding elementary cubes have a common $(d-1)$-dimensional face.

We consider the event

$$
\left\{\exists C_{\infty}^{\varepsilon}\right\},
$$

which is the set of all $\sigma \in \Omega$ for which the graph $G_{\varepsilon}(\sigma)$ contains an infinite cluster, i.e., an infinite connected component. Clearly, if $\varphi$ is a nearest-neighbour potential and $\Phi$ is defined by (2.7) then

$$
\left\{\exists C_{\infty}^{\varepsilon}\right\} \subset\left\{\exists c_{\infty}^{2^{d-1} \varepsilon}\right\}
$$

(the second event was defined in (1.4)).(2.15) shows that low energy site percolation implies low energy bond percolation. We also consider the events

$$
\left\{\exists 1 C_{\infty}^{\varepsilon}\right\} \text {, respectively }\left\{a \in C_{\infty}^{\varepsilon}\right\}
$$


that $G_{\varepsilon}(\cdot)$ contains a unique infinite cluster, respectively that $a \in L^{*}$ belongs to an infinite cluster of $G_{\varepsilon}(\cdot)$. Moreover, when $P$ is an infinite connected subset of $L^{*}$ then we consider the events

$$
\left\{\exists C_{\infty}^{\varepsilon}(P)\right\},\left\{\exists 1 C_{\infty}^{\varepsilon}(P)\right\},\left\{a \in C_{\infty}^{\varepsilon}(P)\right\}
$$

which refer to the restriction of $G_{\varepsilon}(\cdot)$ to $P$.

Now we state our main result.

(2.18) Theorem. Let $P$ be any two-dimensional plane in $L^{*}$ parallel to two of the axes. Fix any $\varepsilon>0$ and $a \in P$. Then

$$
\lim _{\beta \rightarrow \infty} \inf _{\mu \in \mathfrak{G}_{0}(\beta)} \mu\left(a \in C_{\infty}^{\varepsilon}(P)\right)=1
$$

and

$$
\lim _{\beta \rightarrow \infty} \inf _{\mu \in\left(\mathfrak{G}_{0}(\beta)\right.} \mu\left(\exists 1 C_{\infty}^{\varepsilon}(P)\right)=1 .
$$

In particular, for all sufficiently large $\beta$ there is at least one translation invariant Gibbs measure $\mu \in \mathfrak{b}(\beta)$ such that

$$
\mu\left(\exists C_{\infty}^{\varepsilon}\right)=\mu\left(\exists 1 C_{\infty}^{\varepsilon}(P)\right)=1 .
$$

(2.21) Remark. We will actually prove a result which gives more information than (2.20): With probability tending to 1 there exists an infinite low energy cluster $C_{\infty}^{\varepsilon}(P)$ in $P$ which surrounds every finite subset $\Delta$ of $P$ (i.e., $\Delta$ belongs to the interior of a circuit in $\left.C_{\infty}^{\varepsilon}(P)\right)$. In particular, if $d=2$ and $\Phi$ is of the form (2.7) with a nearest neighbour potential $\varphi$, then with probability tending to 1 there is a unique infinite cluster of $g_{\varepsilon}(\cdot)$. (The standard notions of percolation theory such as chain, circuit etc. can be found, for instance, in [4].)

Now let us consider the function

$$
c(\varepsilon, \beta)=\sup _{\mu \in \mathfrak{G}(\beta)} \mu\left(\exists C_{\infty}^{\varepsilon}\right) .
$$

$c(\cdot, \cdot)$ is an increasing function of $\varepsilon$ and only takes the values 0 and 1 ; indeed, if $\mu\left(\exists C_{\infty}^{\varepsilon}\right)>0$ for some $\mu \in \mathfrak{G}(\beta)$ then $\mu\left(. \mid \exists C_{\infty}^{\varepsilon}\right) \in \mathfrak{G}(\beta)$ since $\left\{\exists C_{\infty}^{\varepsilon}\right\}$ is measurable with respect to the tail field

$$
\mathscr{F}_{\infty}=\bigcap_{\Lambda \text { finite } \subset L} \mathscr{F}_{L \backslash \Lambda} .
$$

Thus we obtain

(2.23) Corollary. There is a function $\varepsilon_{c}:[0, \infty[\rightarrow[0, \infty]$ such that for all $\beta \geqq 0$

$$
\begin{array}{ll}
c(\varepsilon, \beta)=0 & \text { when } \varepsilon<\varepsilon_{c}(\beta), \\
c(\varepsilon, \beta)=1 & \text { when } \varepsilon>\varepsilon_{c}(\beta),
\end{array}
$$

and

$$
\varepsilon_{c}(\beta) \rightarrow 0 \quad \text { as } \beta \rightarrow \infty .
$$

The function $\varepsilon_{c}(\beta)$ can be thought of as a quantity which describes how the Gibbs 
measures approach the ground states when the temperature is lowered. If $E$ is finite then $\varepsilon_{c}(\beta)=0$ for sufficiently large $\beta$, whilst $\varepsilon_{c}(\beta)>0$ for all $\beta$ when the conditions of Proposition (1.8) hold. Clearly one would expect that $\varepsilon_{c}(\beta)$ is decreasing or (slightly stronger) that $c(\varepsilon, \beta)$ is an increasing function of $\beta$. In the plane rotator model this monotonicity is also suggested by the Ginibre inequalities [3]; we then could define a threshold temperature $\beta_{c}(\varepsilon)$ which (for this continuous model) should depend continuously on $\varepsilon$, and this would imply that every $\beta$ is critical for the formation of certain infinite clusters, namely for those of $G_{\varepsilon_{c}(\beta)}\left({ }^{\circ}\right)$.

\section{Breaking of Discrete Symmetries}

We continue to consider the setup of the preceding section. To simplify the presentation we assume $d=2$. (In the case $d>2$ we can consider a two-dimensional subsystem.) Now we turn to an investigation of the symmetries of $\Phi$. We let $\mathscr{S}$ denote the class of all measurable transformations

$$
t: E^{v} \rightarrow E^{v}
$$

which satisfy

$$
\Phi_{v}(t s)=\Phi_{v}(s) \quad \text { for all } s \in E^{v}
$$

and belong to the following list of particular transformations:

(i) the reflections $r_{1}, r_{2}$ of $v$ (see (2.4))

(ii) the rotation of $v$

(iii) the transformations of the form $t s=\left(t_{i} s_{i}\right)_{i \in v}\left(s \in E^{v}\right)$ where the $t_{i}: E \rightarrow E$ are measurable mappings which preserve $\lambda$.

Each of these particular transformations has a natural extension to a transformation $T$ of $\Omega$. This is obvious for the reflections and the rotation; if $t$ is of type (iii) then $T$ is given by

$$
T \sigma=\left(t_{j} \sigma_{j}\right)_{j \in L} \quad(\sigma \in \Omega)
$$

where $t_{j}=t_{i}$ if $j \equiv i \bmod 2$ and $\left(t_{i}\right)_{i \in v}=t$.

(Notice that, because of (2.4), both reflections of a type (iii) transformation in $\mathscr{S}$ also belong to $\mathscr{S}$.) In this way each symmetry $t \in \mathscr{S}$ corresponds to a transformation $T$ of $\Omega$ which preserves all measures $\mu \in \mathfrak{G}_{0}(\beta)$.

Now we ask for conditions under which the existence of a unique infinite low energy cluster implies the existence of Gibbs measures in $\mathfrak{5}(\beta)$ for which some of these symmetries are broken. Let $A \subset E^{v}$. For each $\sigma \in \Omega$ we construct a graph $G_{A}(\sigma)$ as follows: The vertices of $G_{A}(\sigma)$ are all sites $a \in L^{*}$ for which

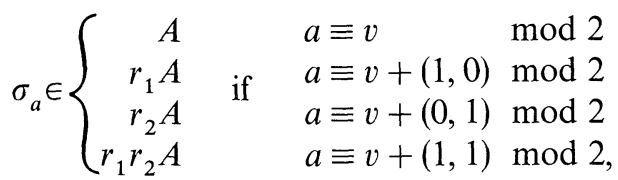


and the edges of $G_{A}(\sigma)$ are all pairs of adjacent vertices. We let

$$
\left\{\exists C_{\infty}^{A}\right\} \text {, respectively }\left\{\exists 1 C_{\infty}^{A}\right\}
$$

denote the events that $G_{A}(\cdot)$ contains an infinite cluster respectively a unique infinite cluster.

Now let $\varepsilon>0$ and suppose the set $\left\{\Phi_{v} \leqq m+\varepsilon\right\} \subset E^{v}$ splits into $N \geqq 2$ disjoint measurable sets $A_{1}, \ldots A_{N}$. (In particular then there are $N$ separated sets of ground states.) We say the splitting is stable if the following condition is satisfied:

Elements $s \in A_{n}$ and $s^{\prime} \in A_{n^{\prime}}$ can coincide on an edge of $v$ (i.e., $s_{i}=s_{i}^{\prime}$ and $s_{j}=s_{j}^{\prime}$ for two adjacent sites $i, j \in v$ ) only if $n=n^{\prime}$.

Of course, this condition implies that each cluster of $G_{\varepsilon}(\sigma)$ is a cluster of $G_{A_{n}}(\sigma)$ for one and only one $n$.

Consequently

$$
\left\{\exists 1 C_{\infty}^{\varepsilon}\right\}=\bigcup_{n=1}^{N} C_{n},
$$

where

$$
C_{n}=\left\{\exists 1 C_{\infty}^{A_{n}}\right\} \backslash \bigcup_{m \neq n}\left\{\exists C_{\infty}^{A_{m}}\right\}
$$

We say the splitting is symmetric if

$$
\text { for all } 1 \leqq n, n^{\prime} \leqq N \text { there is some } t \in \mathscr{S} \text { with } A_{n} \subset t^{-1} A_{n^{\prime}} .
$$

This implies that if $\mu$ is preserved by the symmetries in $\mathscr{S}$ then

$$
\mu\left(C_{1}\right)=\mu\left(C_{2}\right)=\ldots=\mu\left(C_{N}\right) ;
$$

indeed, if $1 \leqq n, n^{\prime} \leqq N$ and $T: \Omega \rightarrow \Omega$ corresponds to the $t$ in (3.6) then

$$
\begin{aligned}
& \left\{\exists 1 C_{\infty}^{\varepsilon}\right\}=T^{-1}\left\{\exists 1 C_{\infty}^{\varepsilon}\right\}, \\
& \left\{\exists C_{\infty}^{A_{n}}\right\} \subset T^{-1}\left\{\exists C_{\infty}^{A_{n}{ }^{\prime}}\right\},
\end{aligned}
$$

and thus

$$
C_{n}=\left\{\exists 1 C_{\infty}^{\varepsilon}, \exists C_{\infty}^{A_{n}}\right\} \subset T^{-1} C_{n^{\prime}} .
$$

In particular, if $\mu \in \mathfrak{G}_{0}(\beta)$ is such that

$$
\mu\left(\exists 1 C_{\infty}^{\varepsilon}\right)>0
$$

then $\mu\left(C_{n}\right)>0$ for all $n$, and the conditional probabilities $\mu_{n}=\mu\left(\cdot \mid C_{n}\right)$ are welldefined and pairwise orthogonal. We would like to have $\mu_{n} \in \mathfrak{G}(\beta)$; this would follow immediately if we had not insisted on the uniqueness of $C_{\infty}^{A_{n}}$ in the definition (3.5) because then the $C_{n}$ would belong to the tail field $\mathscr{F}_{\infty}$. However, $C_{n}$ is invariant under the translations $\theta_{i}, i \equiv 0 \bmod 2$, and therefore (see, for instance, [10]) $\mu$-almost surely equal to a set in $\tilde{F}_{\infty}$. Hence $\mu_{n} \in \mathfrak{G}(\beta)$, and we have proved

(3.7) Theorem. Suppose there is an $\varepsilon>0$ such that

$$
\left\{s \in E^{v}: \Phi_{v}(s) \leqq m+\varepsilon\right\}
$$


splits into $N \geqq 2$ disjoint sets $A_{1}, \ldots, A_{N}$ satisfying (3.3) and(3.6). If $\beta$ is so large that

$$
\mu\left(\exists 1 C_{\infty}^{\varepsilon}\right)>0
$$

for some $\mu \in \mathfrak{b}_{0}(\beta)$ then there are $N$ distinct Gibbs measures $\mu_{1}, \ldots, \mu_{N} \in \mathfrak{5}(\beta)$ which are preserved by the transformations $\theta_{i}, i \equiv 0 \bmod 2$, and are supported respectively by the disjoint sets $C_{1}, \ldots, C_{N}$. In particular, the set ex $\mathfrak{5}(\beta)$ of extreme Gibbs measures consists of at least $N$ elements. Finally, as $\beta \rightarrow \infty$ we have

$$
\mu_{n}\left(\sigma \in \Omega: \sigma_{v} \in A_{n}\right) \rightarrow 1
$$

for all $1 \leqq n \leqq N$.

This last statement is a consequence of (2.19) and (2.20):

$$
\begin{aligned}
& \mu_{n}\left(\sigma: \sigma_{v} \in A_{n}\right) \geqq \mu_{n}\left(v \in C_{\infty}^{A_{n}}\right)=\mu\left(v \in C_{\infty}^{A_{0}}, \exists 1 C_{\infty}^{\varepsilon}\right) / \mu\left(C_{n}\right) \\
& \quad=\left(\frac{1}{N} \mu\left(v \in C_{\infty}^{\varepsilon}, \exists 1 C_{\infty}^{\varepsilon}\right)\right) /\left(\frac{1}{N} \mu\left(\exists 1 C_{\infty}^{\varepsilon}\right)\right) \rightarrow 1 \quad \text { as } \beta \rightarrow \infty
\end{aligned}
$$

when $\mu \in \mathfrak{b}_{0}(\beta)$.

Theorem (3.7) is easily applied to prove the existence of distinct Gibbs measures in various kinds of models. To illustrate this we pick out some well-known examples where symmetry breaking occurs.

(3.8) Discrete Models with Attraction [5,16,17]

Let $E$ be a finite set, $\lambda$ the normalized counting measure, and $\varphi$ a nearest neighbour potential of the form

$$
\varphi(x, y)\left\{\begin{array}{lll}
=m & \\
>m
\end{array} \text { if } \begin{array}{l}
x=y \\
x \neq y
\end{array}\right.
$$

and such that

$$
\varphi(\pi x, \pi y)=\varphi(x, y)
$$

for all $\pi$ in a transitive group of permutations of $E$. (For instance, let $E$ be the group of $N^{\text {th }}$ complex roots of unity and $\varphi(x, y)=-x \cdot y$; the case $N=2$ is the Ising model.) Clearly (3.7) applies and gives $|\operatorname{ex~} \mathfrak{E}(\beta)| \geqq|E|$ when $\beta$ is large.

(3.9) Continuous Extensions of the Above Models

For instance, let $E=S^{1}, \lambda$ be Haar measure, and

$$
\varphi(x, y)=-x \cdot y+\delta\left(\left|x^{N}-1\right|^{2}+\left|y^{N}-1\right|^{2}\right)
$$

for some $\delta>0$ and $N \geqq 2$. For sufficiently small $\varepsilon$ we have $\varphi(x, y) \leqq m+\varepsilon$ iff $x$ and $y$ are close to the same $N^{\text {th }}$ root of unity. Rotational symmetry gives $\mid$ ex $\mathfrak{b}(\beta) \mid \geqq N$ provided $\beta$ is large.

(3.10) Anisotropic Classical Heisenberg Models $[8,14]$

Here $E=S^{N-1}$ (the unit sphere in $\mathbb{R}^{N}$ ) or $E=B^{N}$ (the unit ball in $\mathbb{R}^{N}$ ), $\lambda$ any probability measure which is symmetric with respect to the hyperplane $\left\{x=\left(x_{1}, \ldots, x_{N}\right) \in \mathbb{R}^{N}: x_{1}=0\right\}$ (for instance, the normalized surface or Lebesgue 
measure), and $\varphi$ is a nearest neighbour potential of the form

$$
\varphi(x, y)=-x_{1} y_{1}-\alpha \sum_{k=2}^{N} x_{k} y_{k}
$$

with $0 \leqq \alpha<1$. If $\varepsilon$ is sufficiently small then $\varphi(x, y) \leqq m+\varepsilon$ iff $x_{1}$ and $y_{1}$ are both either close to 1 or -1 . Using the symmetry $x_{1} \leftrightarrow-x_{1}$ we obtain $\mid \operatorname{ex~}(\mathfrak{G}(\beta) \mid \geqq 2$ when $\beta$ is sufficiently large.

\section{(3.11) Unbounded Spin Systems [8]}

In this example, $E$ is the real line and $\lambda$ a mean zero normal distribution; $\varphi$ is a nearest neighbour potential of the form

$$
\varphi(x, y)=(x-y)^{2}+u(x)+u(y)
$$

where $u$ is a double-welled symmetric function such as $u(x)=c_{1} x^{4}-c_{2} x^{2}, c_{1}, c_{2}>0$. $\varphi(x, y)$ is close to its minimum iff $x$ and $y$ fall in the same well of $u$. Thus (3.7) shows that for large $\beta \mid \operatorname{ex}\left(\mathfrak{5}(\beta) \mid \geqq 2\right.$ provided $\mathfrak{G}_{0}(\beta) \neq \varnothing$. But the latter condition is true as can be seen by an obvious modification of the ideas in Sect. 1.5 of Sinai's monograph [17].

(3.12) Shlosman's Model [19]

Here $E=S^{1}, \lambda$ is normalized Haar measure, and $\Phi_{v}: E^{v} \rightarrow \mathbb{R}$ is given by

$$
\Phi_{v}(s)=-\sum_{\substack{i, j \in v \\|i-j|=\sqrt{2}}} s_{i} \cdot s_{j}+J \sum_{\substack{i, j \in v \\|i-j|=1}}\left|s_{i} \cdot s_{j}\right|^{2}
$$

where $J>0$ and $|\cdot|$ denotes Euclidean distance. $\Phi_{v}\left(\begin{array}{cc}s_{4} & s_{3} \\ s_{1} & s_{2}\end{array}\right)$ is minimal iff $s_{1}=s_{3}$, $s_{2}=s_{4}$ and $s_{2}=(0, \pm 1) \cdot s_{1}$. Thus the ground states fall into two separated classes, depending on the sign in the last equality. The transformation

$$
t:\left(\begin{array}{ll}
s_{4} & s_{3} \\
s_{1} & s_{2}
\end{array}\right) \rightarrow\left(\begin{array}{rr}
-s_{4} & s_{3} \\
s_{1} & -s_{2}
\end{array}\right)
$$

belongs to $\mathscr{S}$ and transforms each class into the other. Therefore $\mid \operatorname{ex}(\mathfrak{b}(\beta) \mid \geqq 2$ when $\beta$ is large.

(3.13) The Gertsik-Dobrushin Model $[11,13]$

Let $E=\{-1,1\}$ and suppose $\Phi_{v}: E^{v} \rightarrow \mathbb{R}$ is of the form

$$
\Phi_{v}(s)=-J_{1} \sum_{\substack{i, j \in v \\|i-j|=1}} s_{i} s_{j}-J_{2} \sum_{\substack{i, j \in v \\|i-j|=\sqrt{2}}} s_{i} s_{j}-h \sum_{i \in v} s_{i} .
$$

$\Phi_{v}$ is invariant under reflections and rotations of $v$; in the case $h=0$ the spin reversal $s \rightarrow-s$ also belongs to $\mathscr{S}$. Putting $J_{2}=0$ we obtain the Ising model; in particular, the case $J_{2}=0, J_{1}<0$ corresponds to the Ising antiferromagnet. If $J_{1}=0$ then the model is equivalent to the product of two independent Ising models. For $J_{1} \neq 0$ the ground states of $\Phi_{v}$ are shown in the following phase diagram. 

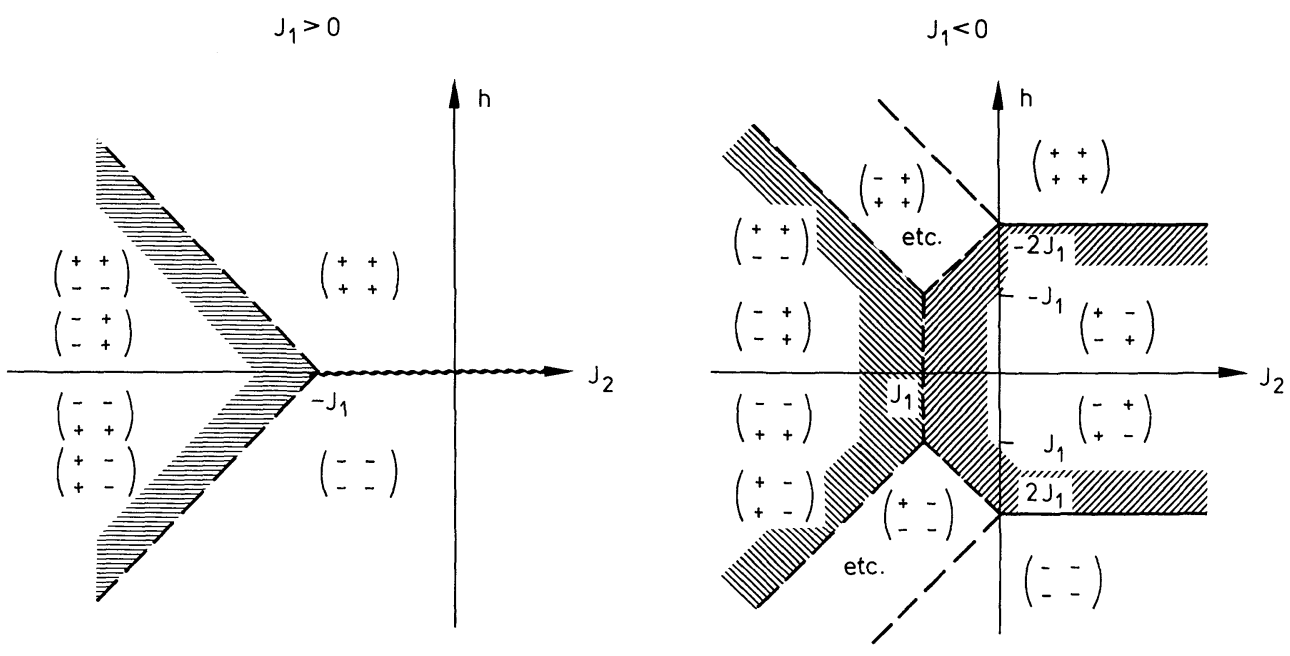

Fig. 1

(The diagram in [13] is erroneous.) The stability condition (3.3) fails in the regions where the ground states consist of an odd number of +'s and -'s and on the broken lines. The symmetry condition (3.6) fails on the separating lines except for the wavy line.

Theorem (3.7) thus applies to the shaded regions and the wavy line and proves the existence of at least two or four distinct Gibbs measures when $\beta$ is sufficiently large. A further model showing a similar phase diagram is the spin version of an eight-vertex model [13]; here

$$
\Phi_{v}(s)=-J_{1} \sum_{\substack{i, j \in v \\|i-j|=1}} s_{i} s_{j}-J_{2} \sum_{\substack{i, j \in v \\|i-j|=\sqrt{2}}}-J_{3} \prod_{i \in v} s_{i} .
$$

The interested reader will find several regions with two or four symmetric ground states satisfying (3.3).

To conclude let us note that Theorem (3.7) is similar to the results of Pirogov and Sinai $[16,17]$ inasmuch as it also gives some information on the typical configurations with respect to the Gibbs measures $\mu_{1}, \ldots, \mu_{N}$; it has the advantage of not being restricted to the case of finite $\mathrm{E}$. But if $E$ is finite then the method of Pirogov/Sinai is more powerful: it works for interactions with arbitrary finite range and does not need a symmetry condition. For example, in the model (3.13) above their method gives the following result: Each point of the solid lines in Fig. 1 has close to it a point for which there are at least three distinct Gibbs measures at low temperatures. However, there is a further possibility to treat such phase transitions without symmetry breaking; this device was described in Sect. 1.6 of part II of [7]. It is not too difficult to see that this method, when it applies, may replace the symmetry argument in the proof of Theorem (3.7). (Recall that (3.6) was only needed in order to deduce from $\mu\left(\exists 1 C_{\infty}^{\varepsilon}\right)>0$ that $\mu\left(C_{n}\right)>0$ for all $1 \leqq n \leqq$. But the same conclusion holds when $\mu\left(v \in C_{\infty}^{\varepsilon} j \exists 1 C_{\infty}^{\varepsilon}\right)$ is close to 1 and $\mu\left(\sigma_{v} \in A_{n}\right)$ is close to $1 / N$ for all $1 \leqq n \leqq N$.) We leave to the reader the precise formulation of the 
theorem obtained in this way, as well as its proof and application to some examples of phase transition without symmetry breaking.

\section{Proofs}

We start with the proof of Theorem (2.18) by considering the Gibbs distribution $\gamma_{A}^{\beta}$ in $\Lambda=\Lambda_{n}$ with periodic boundary condition. The key estimate is formulated in the following

(4.1) Lemma. Suppose for each $a \in \Lambda^{*}$ we are given a measurable function $f_{a}: E^{v} \rightarrow$ $[0, \infty$ [which is invariant under reflections of $v$. Then for all $\beta \geqq 0$ we have

$$
\int \gamma_{\Lambda}^{\beta}(d \sigma) \prod_{a \in \Lambda^{*}} f_{a}\left(\sigma_{a}\right) \leqq \prod_{a \in \Lambda^{*}}\left[\int \gamma_{\Lambda}^{\beta}(d \sigma) \prod_{b \in \Lambda^{*}} f_{a}\left(\sigma_{b}\right)\right]^{1 /|\Lambda|}
$$

Proof. It is well-known $[7,8]$ that $\gamma_{A}^{\beta}$ is reflection positive with respect to the reflections in the pairs of hyperplanes

$$
R_{k}=\left\{x \in \mathbb{R}^{d}: x_{k}=0 \text { or } n\right\}, \quad 1 \leqq k \leqq d .
$$

(To check this it suffices to observe that if the spins on $\Lambda \cap R_{k}$ are fixed then the spin configurations in the two parts of $\Lambda \backslash R_{k}$ are conditionally independent and, up to reflection, identically distributed.) Therefore the chessboard estimates $[7,8]$ apply, and the Lemma follows immediately.

Next we fix an $\varepsilon>0$.

(4.2) Lemma. There is a function $r:[0, \infty[\rightarrow[0,1]$ with the property

$$
r(\beta) \rightarrow 0 \text { as } \beta \rightarrow \infty
$$

such that for all $\beta \geqq 0$ and $\mu \in \mathfrak{F}_{0}(\beta)$ and all finite subsets $D$ of $L^{*}$ the inequality

$$
\mu\left(V_{\varepsilon}(\cdot) \cap D=\varnothing\right) \leqq r(\beta)^{|D|}
$$

holds.

Proof. The set

$$
A=\left\{V_{\varepsilon}(\cdot) \cap D=\varnothing\right\}
$$

is open; hence if $\mu=\lim _{n^{\prime} \rightarrow \infty} \gamma_{\Lambda_{n^{\prime}}}^{\beta}$ for some subsequence $\left(n^{\prime}\right)$ then

$$
\mu(A) \leqq \liminf _{n^{\prime} \rightarrow \infty} \gamma_{\Lambda_{n^{\prime}}}^{\beta}(A) .
$$

Therefore we look for an upper bound of $\gamma_{\Lambda}^{\beta}(A)$ when $\Lambda$ is large enough that $D \subset \Lambda^{*}$. The indicator function of $A$ can be written as

$$
\prod_{a \in D} f\left(\sigma_{a}\right)
$$

where $f: E^{v} \rightarrow\{0,1\}$ is defined by

$$
f(s)=\left\{\begin{array}{lll}
1 & \text { if } & \Phi_{v}(s)>m+\varepsilon \\
0 & & \text { otherwise }
\end{array}\right.
$$




\section{Putting}

$$
f_{a}=\left\{\begin{array}{lll}
f & \text { if } & a \in D \\
1 & & \text { otherwise }
\end{array}\right.
$$

we obtain from (4.1) that

$$
\gamma_{\Lambda}^{\beta}(A) \leqq r_{\Lambda}(\beta)^{|D|}
$$

here

Let us write

$$
r_{\Lambda}(\beta)=\left[\int \gamma_{\Lambda}^{\beta}(d \sigma) \prod_{a \in \Lambda^{*}} f\left(\sigma_{a}\right)\right]^{1 /|\Lambda|}
$$

$$
r_{\Lambda}(\beta)^{|\Lambda|}=I_{\Lambda}^{\beta} / Z_{\Lambda}^{\beta}
$$

By definition

$$
I_{\Lambda}^{\beta}=\int \lambda^{\Lambda}(d \sigma) \exp \left[-\beta H_{\Lambda}(\sigma)\right] \prod_{a \in \Lambda^{*}} f\left(\sigma_{a}\right) \leqq \exp [-\beta|\Lambda|(m+\varepsilon)] .
$$

To find a lower bound for $Z_{\Lambda}^{\beta}$ we let $\varepsilon^{\prime}=\varepsilon / 2$.

The open set

$$
\left\{s \in E^{v}: \Phi_{v}(s)<m+\varepsilon^{\prime}\right\}
$$

contains a nonempty subset of the form

$$
\prod_{i \in v} E_{i}
$$

where the $E_{i}$ are open subsets of $E$; since $\lambda$ is assumed to charge each nonempty open set we have

$$
c=\prod_{i \in v} \lambda\left(E_{i}\right)>0 .
$$

Now consider the event $B$ consisting of all $\sigma \in E^{A}$ which satisfy

$$
\sigma_{j} \in E_{i} \quad \text { if } j \equiv i \bmod 2 \text { and } i \in v .
$$

Then

$$
\lambda^{A}(B) \geqq c^{|\Lambda|}
$$

and

$$
Z_{\Lambda}^{\beta} \geqq \int_{B} \lambda^{\Lambda}(d \sigma) \exp \left[-\beta H_{\Lambda}(\sigma)\right] \geqq \exp \left[-\beta|\Lambda|\left(m+\varepsilon^{\prime}\right)\right] c^{|\Lambda|} .
$$

We thus conclude

$$
r_{\Lambda}(\beta) \leqq r(\beta)=1 \wedge\left(e^{-\beta \varepsilon^{\prime}} / c\right) .
$$

This completes the proof.

Now let $P$ be a fixed plane in $L^{*}$ parallel to two axes. The elements of $P$ are labelled by the sites $u=\left(u_{1}, u_{2}\right) \in \mathbb{Z}^{2}$. Let $Q$ be a subset of $P$ which corresponds to a 
quadrant of $\mathbb{Z}^{2}$. We define

$$
\bar{r}(\beta)=1 \wedge \sum_{\ell \geqq 1} \ell[5 r(\beta)]^{\ell} .
$$

Clearly, $\bar{r}(\beta) \rightarrow 0$ as $\beta \rightarrow \infty$. Thus (2.19) follows from the next

(4.4) Lemma. If $\beta \geqq 0$ and $\mu \in \mathfrak{G}(\beta)$ then

$$
\mu\left(0 \in C_{\infty}^{\varepsilon}(Q)\right) \geqq 1-\bar{r}(\beta) .
$$

Proof. We can write

$$
\left\{0 \notin C_{\infty}^{\varepsilon}(Q)\right\} \subset \bigcup_{D}\left\{V_{\varepsilon}(\cdot) \cap D=\varnothing\right\} ;
$$

here the union extends over all subsets $D$ of $Q$ labelled by finite sequences $\left(u^{(1)}, \ldots, u^{(\ell)}\right)$ in $\mathbb{Z}^{2}$ such that $u_{1}^{(1)}=0, u_{2}^{(\ell)}=0,\left|u^{(k)}-u^{(k+1)}\right| \leqq \sqrt{2}$ for $1 \leqq k<\ell$, and $\left|u^{(k)}-u^{(k+2)}\right|>\sqrt{2}$ for $1 \leqq k<\ell-1$. The number of such sequences of length $\ell$ is less than $\ell 5^{\ell}$. Hence (4.4) follows from (4.2).

Now we investigate the uniqueness of the infinite low energy cluster in $P$. There are several arguments in the literature which prove the uniqueness of infinite clusters; however, these rely on the FKG (Fortuin, Kastelyn and Ginibre) inequalities and a $0-1$ law for tail events. Here we give a different argument which is based on translation invariance. We fix some $\beta$ with $\vec{r}(\beta)<\frac{1}{16}$ and a Gibbs measure $\mu \in \mathfrak{6}_{0}(\beta)$. $\mu$ is invariant under translations. We consider two neighbouring quadrants $Q_{1}$ and $Q_{2}$ in $P$ and the half-plane $\pi=Q_{1} \cup Q_{2}$. For the sake of definiteness we assume

$$
\pi=\left\{u \in \mathbb{Z}^{2}: u_{2} \geqq 0\right\}
$$

Let $A \in \mathscr{F}$ denote the following event: The restriction of $G_{\varepsilon}(\cdot)$ to $\pi$ contains an infinite cluster $C$ such that $(k, 0) \in C$ for infinitely many $k \geqq 0$ and infinitely many $k \leqq 0$. Put

$$
A_{\infty}=\bigcup_{N \geqq 0} \bigcup_{n \geqq N} \theta_{(0, n)} A .
$$

$A_{\infty}$ consists of all $\sigma$ such that for infinitely many $n$ there is an infinite cluster of $G_{\varepsilon}(\sigma) \cap\left\{u \in \mathbb{Z}^{2}: u_{2} \geqq n\right\}$ which meets each of the half-lines $\left\{u_{1} \geqq 0, u_{2}=n\right\}$ and $\left\{u_{1}<0, u_{2}=n\right\}$ infinitely often.

(4.6) Lemma. $\mu\left(A_{\infty}\right) \geqq 1-4 \bar{r}(\beta)$.

Proof. Suppose first

$$
\lambda^{v}\left(\Phi_{v} \leqq m+\varepsilon\right)=1
$$

Then

$$
\mu\left(V_{\varepsilon}(\cdot)=L^{*}\right)=1,
$$

and the assertion is trivially true. Hence we can assume

$$
\lambda^{v}\left(\Phi_{v}>m+\varepsilon\right)>0 .
$$

Poincaré's recurrence theorem $([6]$, Theorem (1.15)) states that

$$
A \subset A_{\infty} \quad \mu \text {-almost surely. }
$$


Therefore we only need to show

$$
\mu(A) \geqq 1-4 \bar{r}(\beta) .
$$

To this end we observe that

where

$$
A \supset A_{+} \cap A_{-} \cap B
$$

$$
\begin{aligned}
& A_{+}=\bigcap_{N \geqq 0} \bigcup_{\ell \geqq N} \theta_{(\ell, 0)} A_{0}, A_{-}=\bigcup_{N \leqq 0} \bigcup_{k \leqq N} \theta_{(k, 0)} A_{0}, \\
& A_{0}=\left\{0 \in C_{\infty}^{\varepsilon}\left(Q_{1}\right)\right\} \cap\left\{0 \in C_{\infty}^{\varepsilon}\left(Q_{2}\right)\right\}
\end{aligned}
$$

and

$$
\begin{gathered}
B=\bigcap_{k<0<\ell} \bigcup_{n \geqq 1} B_{k, \ell, n}, \\
B_{k, \ell, n}=\left\{u \notin V_{\varepsilon}(\cdot) \quad \text { when } k \leqq u_{1} \leqq \ell, u_{2}=n\right\} .
\end{gathered}
$$

Indeed, if $\sigma \in A_{+} \cap A_{-}$then there are infinitely many $k \leqq 0$ and $k \geqq 0$ such that $V_{\varepsilon}(\sigma)$ contains two infinite chains in $\pi$ starting from $(k, 0)$ : one chain going to the left and one chain going to the right. If in addition $\sigma \in B$ then for all $k<\ell$ we have: Each infinite chain in $V_{\varepsilon}(\sigma) \cap \pi$ starting form $(k, 0)$ and going to the right must intersect each infinite chain in $V_{\varepsilon}(\sigma) \cap \pi$ starting from $(\ell, 0)$ and going to the left.

Therefore (4.8) will be proved once we can show

and

$$
\mu\left(A_{+}\right) \geqq 1-2 \bar{r}(\beta), \mu\left(A_{-}\right) \geqq 1-2 \bar{r}(\beta)
$$

$$
\mu(B)=1 \text {. }
$$

(4.9) follows from Poincaré's recurrence theorem since, by Lemma (4.4),

$$
\mu\left(A_{0}\right) \geqq 1-2 \bar{r}(\beta) .
$$

To verify (4.10) it sufficies to show that for all $k<0<\ell$

$$
\mu\left(B_{k, \ell, n} \text { occurs for infinitely many } n \geqq 1\right)=1 \text {. }
$$

Let $F$ denote the complement of the set in this expression and suppose $\mu(F)>0$. Then

$$
v=\mu(\cdot \mid F)
$$

is a well-defined probability measure. $v$ is invariant under $\theta_{(0,1)}$ (Since $\mu$ and $F$ are) and belongs to $(\mathfrak{5}(\beta)$ because $F$ is $\mu$-almost surely tail-measurable (see [10] for a proof). Using again Poincaré's recurrence theorem we see that

$$
v\left(B_{k, \ell, 0}\right)=0 .
$$

To get a contradiction we let $\Lambda$ denote the union of the elementary cubes $u \in P$ with $k \leqq u \leqq \ell, u_{2}=0$. Then $B_{k, \ell, 0} \in \mathscr{F}_{A}$. By definition of $\left(\mathfrak{5}(\beta), v\right.$ is equivalent to $\lambda^{L}$ on $\mathscr{F}_{\Lambda}$. Hence

$$
\lambda^{L}\left(B_{k, \ell, 0}\right)=0
$$


This contradicts (4.7). Indeed, (4.7) implies that the open set $\left\{\Phi_{v}>m+\varepsilon\right\}$ contains an open product set $\prod_{i \in v} E_{i}$ of positive $\lambda$-measure.

Therefore if we define

$$
E_{j}=E_{i} \text { if } j \equiv i \bmod 2
$$

then

$$
0<\lambda^{\Lambda}\left(\prod_{j \in \Lambda} E_{j}\right) \leqq \lambda^{L}\left(B_{k, \ell, 0}\right) .
$$

Next we let $S$ denote the event consisting of all those $\sigma$ for which $V_{\varepsilon}(\sigma) \cap P$ contains an infinite cluster $C(\sigma)$ such that every finite subset of $P$ is surrounded by a circuit in $C(\sigma)$. Clearly

$$
S \subset\left\{\exists 1 C_{\infty}^{\varepsilon}(P)\right\} .
$$

The next Lemma proves (2.20).

(4.12) Lemma. $\mu(S) \geqq 1-16 \bar{r}(\beta)$.

Proof. Let $S_{1}=A_{\infty}$ and define $S_{2}, S_{3}, S_{4}$ as the events which are obtained from $A_{\infty}$ by a rotation of the plane $P$. Then

$$
S_{1} \cap S_{2} \cap S_{3} \cap S_{4} \subset S
$$

and (4.12) follows from (4.6).

To complete the proof of Theorem (2.18) we observe that if $\mu \in \mathfrak{G}_{0}(\beta)$ and

$$
\mu\left(\exists 1 C_{\infty}^{\varepsilon}(P)\right)>0
$$

then

$$
v=\mu\left(\cdot \mid \exists 1 C_{\infty}^{\varepsilon}(P)\right)
$$

is a translation invariant measure in $(5(\beta)$; this is seen by the same argument as in the proof of (4.10).

Finally we turn to the proof of Proposition (1.8). We consider the sublattice

$$
\tilde{L}^{*}=\left\{a \in L^{*}: a \equiv v \bmod 2\right\}
$$

of $L^{*}$. The elementary cubes $a \in \tilde{L}^{*}$ form a partition of $L$. Let

$$
\widetilde{\Omega}=\{0,1\} \tilde{L}^{*} .
$$

$\widetilde{\Omega}$ is endowed with the usual $\sigma$-algebra. For each $\varepsilon>0$ we define a measurable mapping

$$
T_{\varepsilon}: \Omega \rightarrow \widetilde{\Omega}
$$

as follows: If $\sigma \in \Omega$ and $a \in \tilde{L}^{*}$ then we put $\left(T_{\varepsilon} \sigma\right)_{a}=1$ if $a$ contains a pair $\{i, j\}$ of adjacent sites of $L$ with $\varphi\left(\sigma_{i}, \sigma_{j}\right) \leqq m+\varepsilon$; otherwise we put $\left(T_{\varepsilon} \sigma\right)_{a}=0$. For each $\omega \in \widetilde{\Omega}$ we define a graph $\widetilde{G}(\omega)$ : the vertex set is

$$
\tilde{V}(\omega)=\left\{a \in \tilde{L}^{*}: \omega_{a}=1\right\},
$$

and the set of edges consists of all pairs of *adjacent sites of $\tilde{V}(\omega)$. Here $a, b \in \tilde{L}^{*}$ are said to be *adjacent if $a=b+2 u$ for some $u=\left(u_{1}, \ldots, u_{d}\right)$ with $u_{k} \in\{-1,0,1\}$ 
for all $1 \leqq k \leqq d$. It is not difficult to see that if $\sigma \in \Omega$ and $c(\sigma)$ is an infinite cluster of $g_{\varepsilon}(\sigma)$ then

$$
C=\left\{a \in \tilde{L}^{*}: a \cap c(\sigma) \text { contains an edge }\right\}
$$

is an infinite connected subset of $\tilde{G}\left(T_{\varepsilon} \sigma\right)$. In other words:

$$
\left\{\exists c_{\infty}^{\varepsilon}\right\} \subset T_{\varepsilon}^{-1}\left\{\exists \tilde{C}_{\infty}\right\} ;
$$

here $\left\{\exists \widetilde{C}_{\infty}\right\}$ is used to denote the event that $\widetilde{G}(\cdot)$ contains an infinite cluster. For $\mu \in \mathfrak{G}(\beta)$ we let $\tilde{\mu}_{\varepsilon}=T_{\varepsilon}(\mu)$ denote its image under $T_{\varepsilon}$. (4.14) gives

$$
\mu\left(\exists c_{\infty}^{\varepsilon}\right) \leqq \tilde{\mu}_{\varepsilon}\left(\exists \tilde{C}_{\infty}\right) .
$$

If $\beta=0$ then $\tilde{\mu}_{\varepsilon}$ is a product measure of the form

$$
\tilde{v}_{p}=(1-p, p)^{\tilde{L}^{*}} \text {. }
$$

It is well-known [18] that there is a percolation threshold $p_{c}>0$ such that

$$
\tilde{v}_{p}\left(\exists \widetilde{C}_{\infty}\right)=0 \quad \text { when } p<p_{c} .
$$

Thus our problem reduces to finding conditions on $\varepsilon$ and $\beta$ which would imply

for some $p<p_{c}$.

$$
\tilde{\mu}_{\varepsilon}\left(\exists \tilde{C}_{\infty}\right) \leqq \tilde{v}_{p}\left(\exists \tilde{C}_{\infty}\right)
$$

It is easily seen that (4.17) holds whenever $\tilde{\mu}_{\varepsilon}<\tilde{v}_{p}$ : here " $\prec$ " is the ordering between probability measures on $\tilde{\Omega}$ which is defined as follows: $\tilde{v}<\tilde{v}^{\prime}$ iff for each increasing function $f$ on $\widetilde{\Omega}$ which depends only on finitely many coordinates

$$
\int f d \tilde{v} \leqq \int f d \tilde{v}^{\prime} .
$$

By means of Holley's inequalities [2] we obtain the following Lemma which, together with (4.15) and (4.16), completes the proof of Proposition (1.8).

(4.18) Lemma. Suppose the hypotheses of Proposition (1.8) are satisfied. Then there exists a decreasing function $\beta(\cdot):[0, \infty[\rightarrow[0, \infty[$ such that

$$
\beta(\varepsilon) \rightarrow \infty \text { as } \varepsilon \rightarrow 0
$$

and

$$
\tilde{\mu}_{\varepsilon}<\tilde{v}_{p} \text { for some } p<p_{c}
$$

whenever $\varepsilon>0,0 \leqq \beta<\beta(\varepsilon)$ and $\mu \in \mathfrak{G}(\beta)$.

Proof. Holley's theorem [2] asserts that if $V$ is any finite set and $\tilde{v}_{V}$ and $\tilde{v}_{V}^{\prime}$ are two probability measures on $\{0,1\}^{V}$ satisfying

$$
\tilde{v}_{V}(\omega \wedge \zeta) \tilde{v}_{V}^{\prime}(\omega \vee \zeta) \geqq \tilde{v}_{V}(\omega) \tilde{v}_{V}^{\prime}(\zeta)
$$

for all $\omega, \zeta \in\{0,1\}^{V}$ then

$$
\int f d \tilde{v}_{V} \leqq \int f d \tilde{v}_{V}^{\prime}
$$

for each increasing function $f$ on $\{0,1\}^{V}$. If $\tilde{v}_{V}^{\prime}=(1-p, p)^{V}$ (the product measure) and $r=p /(1-p)$ then $(4.19)$ reduces to the requirement

$$
\tilde{v}_{V}(\omega) \leqq r^{|\omega|-|\zeta|} \tilde{v}_{V}(\zeta)
$$


for all $\zeta \leqq \omega$; here

$$
|\omega|=\sum_{a \in V} \omega_{a}
$$

Therefore we have to look for conditions on $\varepsilon$ and $\beta$ which would imply (4.20) for some $r<r_{c}=p_{c} /\left(1-p_{c}\right)$ whenever $V$ is a finite subset of $\tilde{L}^{*}, \mu \in \mathfrak{b}(\beta)$, and $\tilde{v}_{V}$ is the projection of $\tilde{\mu}_{\varepsilon}$ onto $\{0,1\}^{V}$. We fix two configurations $\omega, \zeta \in\{0,1\}^{V}$ with $\zeta \leqq \omega$. Let

$$
V_{1}=\left\{a \in V: \zeta_{a}<\omega_{a}\right\}, V_{2}=\left\{a \in V: \zeta_{a}=\omega_{a}\right\},
$$

and let $\Lambda$ denote the union of the elementary cubes $a \in V_{1}$. Define

$$
\left.\begin{array}{ll}
A_{0}=\left\{\sigma \in \Omega:\left(T_{\varepsilon} \sigma\right)_{a}=0\right. & \text { for all } \left.a \in V_{1}\right\} \\
A_{1}=\left\{\sigma \in \Omega:\left(T_{\varepsilon} \sigma\right)_{a}=1\right. & \text { for all } a \in V_{1}
\end{array}\right\}
$$

and

Then

$$
B=\left\{\sigma \in \Omega:\left(T_{\varepsilon} \sigma\right)_{a}=\omega_{a} \text { for all } a \in V_{2}\right\}
$$

and

$$
\tilde{v}_{V}(\zeta)=\int_{B} \mu(d \sigma) \gamma_{A}^{\beta}\left(A_{0} \mid \sigma\right)
$$

$$
\tilde{v}_{V}(\omega)=\int_{B} \mu(d \sigma) \gamma_{A}^{\beta}\left(A_{1} \mid \sigma\right)
$$

Therefore (4.20) holds whenever for each $\sigma \in \Omega$

$$
\int_{A_{1}} \lambda^{L}(d \tau) \exp \left[-\beta H_{\Lambda}(\tau \mid \sigma)\right] \leqq r^{|\omega|-|\zeta|} \int_{A_{0}} \lambda^{L}(d \tau) \exp \left[-\beta H_{\Lambda}(\tau \mid \sigma)\right]
$$

By hypothesis, $|\varphi| \leqq M$ for some constant $M<\infty$. Thus

$$
\left|H_{A}(\tau \mid \sigma)\right| \leqq(|\omega|-|\zeta|) \bar{M}
$$

where $\bar{M}=(2 d) 2^{d} M$, and (4.21) holds provided

Let

$$
\lambda^{L}\left(A_{1}\right) \leqq\left(\mathrm{re}^{-2 \beta \bar{M}}\right)^{|\omega|-|\zeta|} \lambda^{L}\left(A_{0}\right)
$$

$$
p(\varepsilon)=\lambda^{v}\left(s \in E^{v}: \varphi\left(s_{i}, s_{j}\right) \leqq m+\varepsilon \text { for two adjacent sites } i, j \in v\right) .
$$

Then

$$
\lambda^{L}\left(A_{1}\right)=p(\varepsilon)^{|\omega|-|\zeta|}, \lambda^{L}\left(A_{0}\right)=(1-p(\varepsilon))^{|\omega|-|\zeta|} .
$$

Hence (4.20) holds for some $r<r_{c}$ whenever

$$
r(\varepsilon)=p(\varepsilon) /(1-p(\varepsilon))<r_{c} e^{-2 \beta \bar{M}}
$$

By hypothesis,

$$
r(\varepsilon) \downarrow 0 \quad \text { as } \quad \varepsilon \downarrow 0 \text {. }
$$

Thus we conclude that

$$
\beta(\varepsilon)=(1 / 2 \bar{M}) \log _{+}\left(r_{c} / r(\varepsilon)\right)
$$

is a function with the required properties. 
Acknowledgement. I am indebted to S. Miracle-Sole for suggesting the study of "small deviation clusters" in the plane rotator model.

\section{References}

1. Aizenman, M. : Commun, Math. Phys. 73, 83-94 (1980)

2. Batty, C. J. K., Bollmann, H. W. : Z. Wahrsch. Verw. Gebiete 53, 157-173 (1980)

3. Bricmont, J., Fortaine, J. R., Landau, L. J. : Commun. Math. Phys. 56, 281-296 (1977)

4. Coniglio, A., Nappi, C. R., Peruggi, F., Russo, L. : Commun. Math. Phys. 51, 315-323 (1976)

5. Dobrushin, R. L. : Functional Anal. Appl. 2, 302-312 (1968)

6. Friedman, N. A. : Introduction to ergodic theory. New York : 1970 van Nostrand Reinhold

7. Fröhlich, J., Israel, R., Lieb, E. H., Simon, B. : Commun. Math. Phys. 62, 1-34 (1978); J. Stat. Phys. 22, 297-347 (1980)

8. Fröhlich, J., Lieb, E. H. : Commun. Math. Phys. 60, 233-267 (1978)

9. Fröhlich, J., Simon, B., Spencer, T. : Commun. Math. Phys. 50, 79-85 (1976)

10. Georgii, H. O. : Commun. Math. Phys. 32, 107-118 (1973)

11. Gertsik, V. M., Dobrushin, R. L. : Funct. Anal. Appl. 8, 201-211 (1974)

12. Higuchi, Y. : In :Proc. Colloquium on Random Fields (Esztergom, June 1979) (to appear)

13. Holsztynski, W., Slawny, J. : Commun. Math. Phys. 61, 177-190 (1978)

14. Malyshev, V. A. : Commun. Math. Phys. 40, 75-82 (1975)

15. Messager, A., Miracle-Solé, S., Pfister, C. : Commun. Math. Phys. 58, 19-29 (1978)

16. Pirogov, S. A., Sinai, Ya. G. : Theor. Math. Phys. 25, 1185-1192 (1975); 26, 39-49 (1976)

17. Sinai, Ya. G. : Theory of phase transitions. Rigorous results (in press)

18. Shante, V. K. S., Kirkpatrick, S. : Adv. Phys. 20, 325-357 (1971)

19. Shlosman, S. B. : Commun. Math. Phys. 71, 207-212 (1980)

Communicated by E. Lieb

Received October 27, 1980; in revised form January 19, 1981 
